Check for updates

Cite this: DOI: $10.1039 / \mathrm{c7cc07306b}$

Received 18th September 2017

Accepted 15th December 2017

DOI: $10.1039 / c 7 c c 07306 b$

rsc.li/chemcomm

\section{Polymersomes with asymmetric membranes and self-assembled superstructures using pentablock quintopolymers resolved by electron tomography $\dagger$}

\author{
J. S. Haataja, (D) ${ }^{a}$ N. Houbenov, (D)*a V. Aseyev, (D) ${ }^{b}$ P. Fragouli, ${ }^{c}$ H. latrou, (D) \\ R. Sougrat, ${ }^{d}$ N. Hadjichristidis (D) *d and O. Ikkala (D) *a
}

Polystyrene-block-poly(1,4-isoprene)-block-poly(dimethyl siloxane)block-poly(tert-butyl methacrylate)-block-poly(2-vinyl pyridine), PS- $b$-PI- $b$-PDMS- $b$-PtBMA- $b$-P2VP, self-assembles in acetone into polymersomes with asymmetric (directional) PI-b-PDMS membranes. The polymersomes, in turn, self-assemble into superstructures. Analogically to supravesicular structures at a smaller length scale, we refer to them as suprapolymersome structures. Electron tomograms are shown to be invaluable in the structural assessment of such complex self-assemblies.

Block copolymers allow progressively ever more complex selfassemblies upon involving an increasing number of constituent blocks, ${ }^{1}$ where the structures depend on the block lengths, block-block and block-solvent interactions, interface curvatures, and molecular topologies. In the simplest case, diblock copolymers provide micellar or polymersome (vesicular-like) self-assemblies in solutions. ${ }^{2}$ However, more complex and hierarchical solutionbased self-assemblies are pursued, e.g., for nanochemistry, encapsulation, and biological applications. ${ }^{3}$ Use of three different blocks, i.e., linear or star-shaped $\mathrm{ABC}$ triblock terpolymers, provides micellar, multicompartmentalized, or patchy colloidal particles and their superstructures in solvent media. ${ }^{4}$ Mastering the solution-based self-assembly becomes challenging already therein due to the many interlinked parameters affecting the final structure. In addition, the self-assembly becomes particularly dependent on the specific selection and order of application of solvents. ${ }^{4 k, p, s}$ Non-equilibrium (intermediate) phases can also become kinetically trapped. ${ }^{4 d, e}$ By incorporating four or more

\footnotetext{
${ }^{a}$ Department of Applied Physics, Aalto University, FI-00076, Aalto, Espoo, Finland. E-mail: nikolay.houbenov@aalto.fi, olli.Ikkala@aalto.fi

${ }^{b}$ Department of Chemistry, University of Helsinki, P.O. Box 55, FI-00014 Helsinki, Finland

'Department of Chemistry, University of Athens, Panepistimiopolis, Zografou, 15771, Athens, Greece

${ }^{d}$ King Abdullah University of Science and Technology (KAUST), Thuwal 23955-6900, Kingdom of Saudi Arabia. E-mail: Nikolaos.Hadjichristidis@kaust.edu.sa

$\dagger$ Electronic supplementary information (ESI) available: Experimental details and additional data. See DOI: 10.1039/c7cc07306b
}

blocks, even more advanced structures are foreseen but at the same time the phase space to design the microphase separation becomes dauntingly large. In addition, selection of solvents and processing become increasingly subtle. Block copolymers with four or five blocks have been synthesized and examples of the complexity of their self-assemblies have been indicated in some cases. ${ }^{4 q, 5}$ Another emerging generic challenge, equally important and often not fully appreciated, is related to how to identify the microphase domains for complex multiblock copolymer structures using electron microscopies, as the number of staining agents and their selectivity are limited. We address these aspects in this article with a model polymer.

Here we report complex solution self-assembly of a pentablock quintopolymer, polystyrene-block-poly(1,4-isoprene)-blockpoly(dimethyl siloxane)-block-poly(tert-butyl methacrylate)-blockpoly(2-vinyl pyridine), i.e., PS- $b$-PI- $b$-PDMS- $b$-PtBMA- $b$-P2VP (Fig. 1). The polymer is synthesized by sequential anionic polymerization and selective linking, ${ }^{5 h}$ with molecular weights being 32.4, 13.1, 24.7, 50.0, and 41.7 kDa for the PS, PI, PDMS, PtBMA, and P2VP blocks and a narrow polydispersity index (PDI $=1.05)$. We hypothesized that such a block copolymer is a relevant model for complex polymersomes for the following reasons: no solvent exists that is equally good for all of the blocks. Here, the central blocks (PI and PDMS) have a different solubility behavior than the end blocks (PS, PtBMA, and P2VP). We selected acetone as a

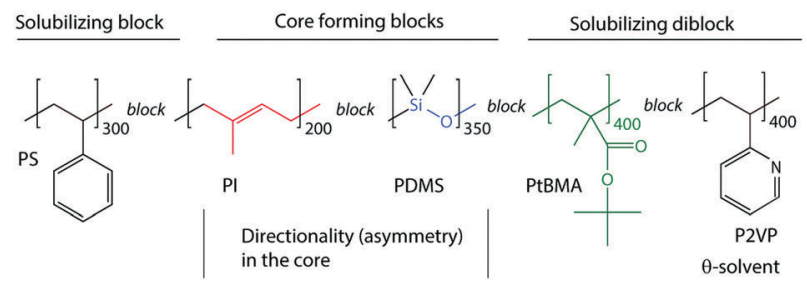

Fig. 1 The chemical structure of the pentablock quintopolymer, and the number of repeat units within the blocks (PDI 1.05). The solubilizing blocks in acetone are indicated, whereas the PI- $b$-PDMS blocks forming the asymmetric membrane are insoluble. The less soluble P2VP-block is suggested to provide the cohesion to form suprapolymersome superstructures. 
solvent (see the ESI $\dagger$ ) as it leads to collapse of the PI and PDMS blocks and it is selective to the end blocks. A subtlety exists as P2VP is less soluble in acetone (theta solvent) than PtBMA. Due to the amphiphilicity, an asymmetric self-assembly into polymersomes is expected with solubilizing PS blocks on one side and PtBMA/P2VP on the other side of the PI/PDMS core. The PI/PDMS core blocks would form asymmetric (directional) membranes (Fig. 1 and 5). Importantly, the asymmetricity would prevent "back looping" within the polymersome core domain, in contrast to e.g. ABA triblock terpolymers.

Indeed, transmission electron microscopy (TEM) showed colloidal scale objects with sizes $d \sim 100-300 \mathrm{~nm}$, and $\mathrm{OsO}_{4}$ staining revealed their complex internal hierarchical structures involving well-defined shell-like substructures of $30-50 \mathrm{~nm}$ sizes (Fig. 2a and b). Obviously, the TEM micrographs indicate polymersomes that are fused into higher level superstructures. For additional data, see Fig. S3-S8 for cryo-TEM and dynamic light scattering (DLS) in the ESI. $\dagger$ Several questions arise: how to identify the internal microphases and why are the constituent polymersomes fused into superstructures? A conventional approach is to adopt different stainings to identify the phases. However, as the number of blocks is increased, achievement of contrast becomes increasingly difficult. ${ }^{6}$ Therefore, the challenges of the present work also reflect those of future complex self-assemblies in more general.

Starting from the unstained samples dried from acetone, the internal structure is rather featureless (Fig. 3a), with a hint of bright spots of diameter around $5 \mathrm{~nm}$.

The assignment of block copolymeric microdomains in TEM micrographs is occasionally done quantitatively, i.e., by

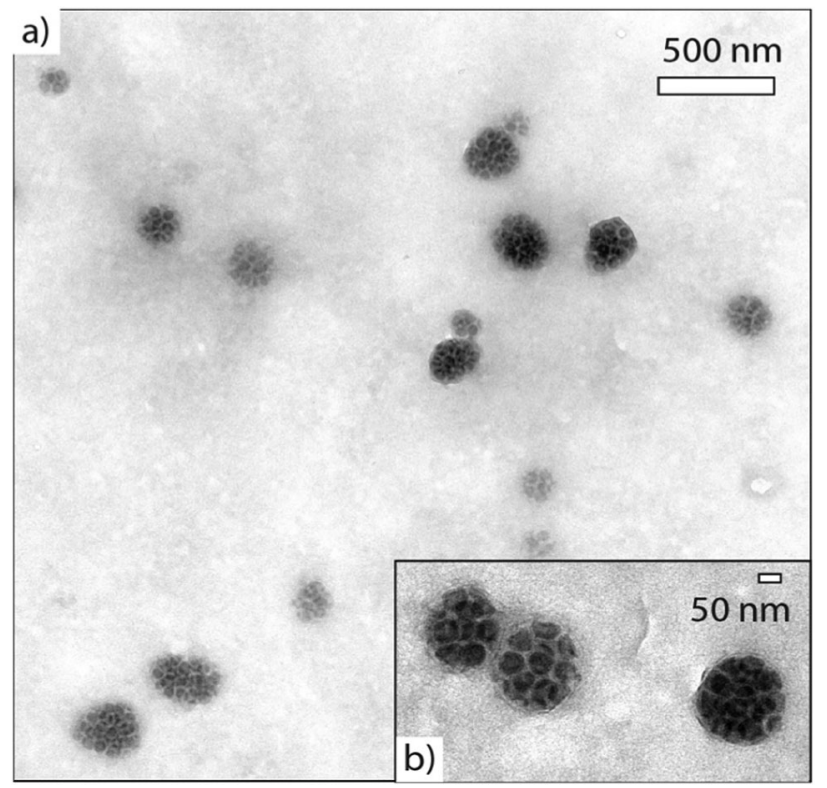

Fig. 2 The self-assembled structures of pentablock quintopolymer PS- $b$ PI- $b$-PDMS- $b$-PtBMA- $b$-P2VP drop cast from acetone, resolved by TEM. (a) Overall low magnification micrograph, illustrating the superstructures and their size distribution. (b) Larger magnification micrograph, showing the internal structure. Samples were stained with $\mathrm{OsO}_{4}$.

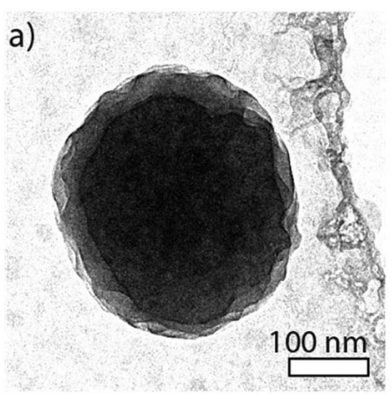

b)

c)

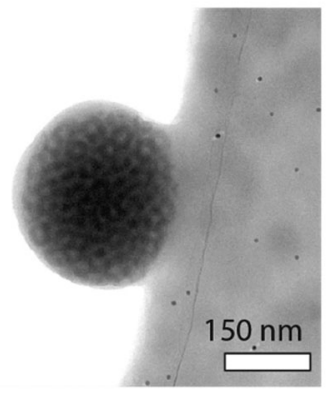

d)
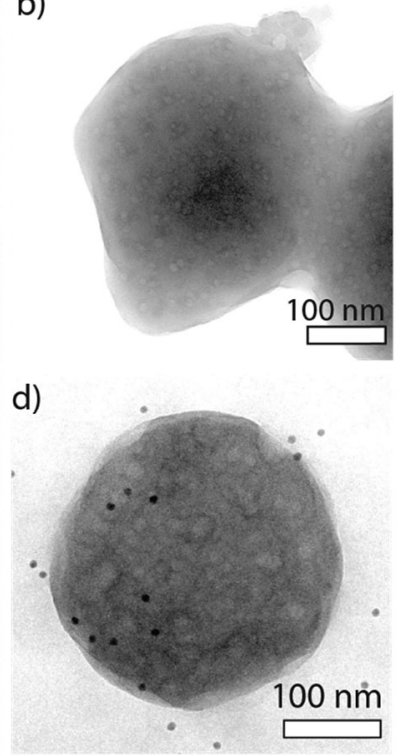

Fig. 3 Resolving the internal microphases by staining upon casting from acetone: (a) without staining, (b) $\mathrm{I}_{2}$-staining, (c) $\mathrm{OsO}_{4}$-staining, and (d) $\mathrm{RuO}_{4}$-staining. The small black beads in (c) and (d) are fiducial markers used for tomographic purposes. The existence of assemblies also in solution is confirmed by cryo-TEM measurements (cf. ESI, $\uparrow$ Fig. S3).

comparing the electron densities. ${ }^{4 h}$ Since the contrast mechanism in bright-field TEM is mainly dependent on scattering properties rather than electron density ${ }^{7}$ (see the ESI $\uparrow, 3.3$ ), we used molecular dynamics (MD) simulations and the multislice approach to obtain quantitative estimates on the expected intensities of the different polymers present in TEM (see the ESI $\dagger$, 3.4). It turns out that there exists no clear discernible contrast between different blocks, or at least not between blocks that are amorphous and have the bulk density of the corresponding homopolymer. Therefore it is not surprising that we do not observe clear contrast differences, except for the brighter spots, which might be accounted for by beam damage or swelling of solvophilic blocks before drying.

To reveal the internal structure, various stainings are incorporated. First, the dried samples were exposed to an $\mathrm{I}_{2}$-atmosphere for $20 \mathrm{~min}$, where $\mathrm{I}_{2}$ selectively absorbs to the P2VP domains. ${ }^{8}$ No clear improvement in contrast was observed (even for longer staining times or when $\mathrm{CH}_{3} \mathrm{I}$ was alternatively used). Still, surprisingly, the initially approximately spherical PS- $b$-PI- $b$ PDMS- $b$-PtBMA- $b$-P2VP superstructures showed spreading onto a carbon-coated grid (Fig. 3b). This suggests that exposure to $I_{2}$ modifies the cohesion of the matrix of the superstructures. This suggests that P2VP belongs to the connective matrix of the superstructures.

$\mathrm{OsO}_{4}$-staining leads to the highest contrast within the superstructures. Accordingly, the internal structure of the individual particles in acetone is composed of dark shells $(d \sim 20 \pm$ $10 \mathrm{~nm}$ ) connected by an unstained polymer matrix, see Fig. 3c and $4 \mathrm{~b}$. Since $\mathrm{OsO}_{4}$ reacts with unconjugated double bonds (found in PI), it is safe to conclude that PI is confined to the polymersome membranes. ${ }^{9}$ 
Next we will examine $\mathrm{RuO}_{4}$-staining. $\mathrm{RuO}_{4}$ is an aggressive chemical used to selectively stain PS, P2VP, and P4VP and also the interfaces of PDMS block copolymers. ${ }^{8 b, 10}$ Such a diverse behaviour is explained sooner by the diffusion and accumulation rates of $\mathrm{RuO}_{4}$ in the polymer microdomains rather than by chemical selectivity. ${ }^{6 a}$ This makes it challenging to achieve selective staining also in our system. Fig. 3d shows a $\mathrm{RuO}_{4}$-stained sample, where faint rings are seen. The maximum diameter of these rings does not appear to exceed the diameter of the ones observed in $\mathrm{OsO}_{4}$-stained samples. This, in turn, suggests that there must be a region near the interface of PI in which $\mathrm{RuO}_{4}$ accumulates.

Due to challenges in characterizing self-assembled systems from 2D TEM projections, electron tomographic (ET) reconstructions have turned out to be invaluable. ${ }^{6 b-h}$ By ET (Fig. 4) we confirm that the superstructures are composed of several spherical core-shell ("onion-like") subunits $(d \sim 55 \pm 13 \mathrm{~nm})$. By calculating the radial distribution functions (RDFs) from several subunits for both unstained and stained samples we can quantitatively estimate the structure of the subunits (Fig. S7, ESI $\dagger$ ). Again, starting from the unstained samples (Fig. 3a) we confirm that the core regions of the subunits are composed of hollow or low-density pockets with radii less than $5 \mathrm{~nm}$ surrounded by darker shells with thicknesses of 3-5 $\mathrm{nm}$. These pockets suggest that there are solvent rich regions that form upon drying, which implies polymersome-like instead of micellar structures. Radial density functions from $\mathrm{OsO}_{4}$-stained samples showed an increase in the radii of the pockets from $<5 \mathrm{~nm}$ to $<10 \mathrm{~nm}$ and that the PI-shell covers a region roughly from $15 \mathrm{~nm}$ to $25 \mathrm{~nm}$ from the centre. Comparison to the $\mathrm{RuO}_{4}$-stained samples indicated two concentric rings of radii of 8 and $20 \mathrm{~nm}$, respectively, with thicknesses of few nanometers. Staining with $\mathrm{RuO}_{4}$ and consequently with $\mathrm{OsO}_{4}$ causes a contraction of the outer ring from 20 to $15 \mathrm{~nm}$. Further staining with either $\mathrm{I}_{2}$ or $\mathrm{CH}_{3} \mathrm{I}$ did not provide additional information. A probable cause for these shifts is the swelling of appropriate polymer phases upon staining.

Based on these observations and tomograms ( $c f$. Fig. 4 and 5a), the suggested polymersome superstructures are schematically shown in Fig. $5 \mathrm{~b}$ and c. The connective matrix is P2VP, as

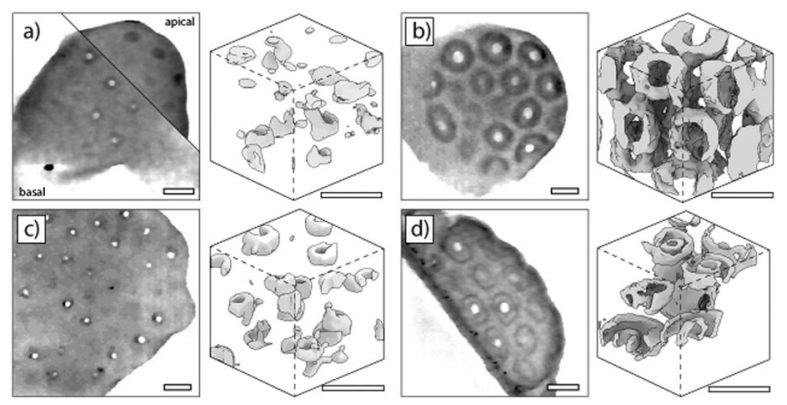

Fig. 4 Electron tomogram (ET) reconstructions from differently stained and unstained samples in acetone, as illustrated using isosurface presentations of segmented volumes: (a) without staining, (b) $\mathrm{OsO}_{4}$ staining, (c) $\mathrm{I}_{2}$ staining, and (d) $\mathrm{RuO}_{4}$ staining. Cross-sections from the original reconstructions are also shown as a reference. The scale bars indicate $50 \mathrm{~nm}$. Note that in (d) a deformed and thinner superstructure was used for imaging of the internal structure, where better image quality was obtained. a)

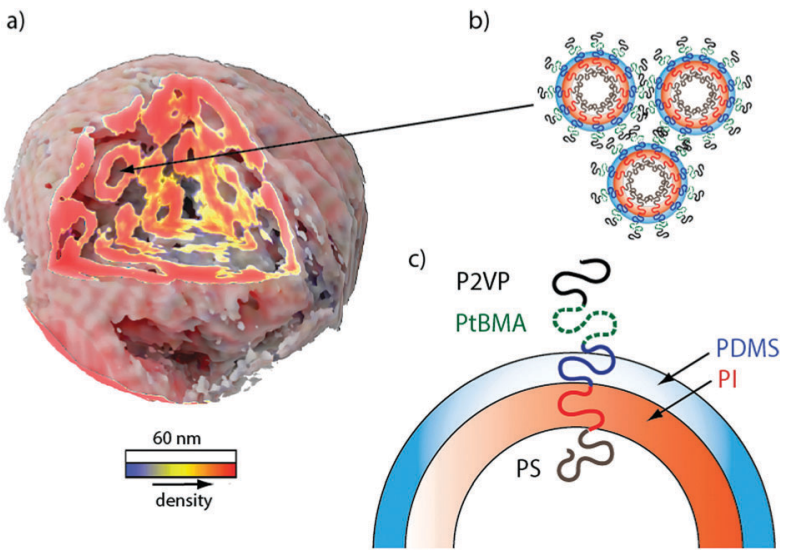

Fig. 5 (a) $\mathrm{OsO}_{4}$-stained tomogram of a PS- $b$-PI- $b$-PDMS- $b$-PtBMA- $b$ P2VP polymersome superstructure in acetone. PI-shells are most preferably stained. The connective matrix is omitted in the volumetric illustration. (b and c) Schematic of the suggested internal microphase separated structure. The slightly lower solubility of P2VP in acetone suggests promoted adhesion between the internal polymersome substructures to provide their superstructures.

shown in Fig. 3b. Acetone is a theta-solvent for P2VP, thus capable of providing slight adhesion between the constituent polymersomes by borderline collapse. The next layer is PtBMA, which dissolves well in acetone, being a good solubilising block. The core membrane is asymmetrically formed by the PI and PDMS shells. The innermost microdomain consists of PS as a solubilising block. Based on this suggestion, each block has a function, and spontaneously formed polymersome superstructures are suggested. In analogy to supravesicular structures at an order of magnitude smaller length scale, ${ }^{11}$ the present structures could be claimed as the first reported suprapolymersomic structures.

In conclusion, we have shown that pentablock quintopolymer PS- $b$-PI- $b$-PDMS- $b$-PtBMA- $b$-P2VP self-assembles into polymersome superstructures, i.e. suprapolymersomes. The polymersome membranes are asymmetric, which suggests conceptually new routes to drive directional transport through the asymmetric (Janus-like) polymersome membranes. We foresee that by careful design of multiblock copolymers highly complex superstructures with controlled internal structures will be achieved but at the same time their characterization will also become increasingly difficult. The present work therefore illustrates some of the general future challenges when multiblock copolymeric self-assemblies are approached. We also propose combination of 2D HR-TEM and 3D ET for the identification of the microphases of complex selfassemblies.

J. S. Haataja, N. Houbenov and O. Ikkala acknowledge the Academy of Finland and ERC (MIMEFUN, project number 291364) for funding. R. Sougrat and N. Hadjichristidis acknowledge the support from the King Abdullah University of Science and Technology (KAUST). This work made use of the Aalto University Nanomicroscopy Center (Aalto-NMC) premises.

\section{Conflicts of interest}

The authors declare no conflicts of interest. 


\section{Notes and references}

1 F. S. Bates, M. A. Hillmyer, T. P. Lodge, C. M. Bates, K. T. Delaney and G. H. Fredrickson, Science, 2012, 336, 434.

2 (a) L. Zhang and A. Eisenberg, Science, 1995, 268, 172; (b) B. M. Discher, Y.-Y. Won, D. S. Ege, J. C.-M. Lee, F. S. Bates, D. E. Discher and D. A. Hammer, Science, 1999, 284, 1143; (c) D. E. Discher and A. Eisenberg, Science, 2002, 297, 967; (d) S. Jain and F. S. Bates, Science, 2003, 300, 460; (e) E. B. Zhulina, M. Adam, I. LaRue, S. S. Sheiko and M. Rubinstein, Macromolecules, 2005, 38, 5330; $(f)$ A. Blanazs, J. Madsen, G. Battaglia, A. J. Ryan and S. P. Armes, J. Am. Chem. Soc., 2011, 133, 16581; (g) J. Zhu, S. Zhang, K. Zhang, X. Wang, J. W. Mays, K. L. Wooley and D. J. Pochan, Nat. Commun., 2013, 4, 2297; (h) R. J. R. W. Peters, M. Marguet, S. Marais, M. W. Fraaije, J. C. M. van Hest and S. Lecommandoux, Angew. Chem., Int. Ed., 2014, 53, 146.

3 (a) X. Wang, G. Guerin, H. Wang, Y. Wang, I. Manners and M. A. Winnik, Science, 2007, 317, 644; (b) H. Qiu, Z. M. Hudson, M. A. Winnik and I. Manners, Science, 2015, 347, 1329.

4 (a) D. J. Pochan, Z. Chen, H. Cui, K. Hales, K. Qi and K. L. Wooley, Science, 2004, 306, 94; (b) N. Hadjichristidis, H. Iatrou, M. Pitsikalis, S. Pispas and A. Avgeropoulos, Prog. Polym. Sci., 2005, 30, 725; (c) S. Kubowicz, J.-F. Baussard, J.-F. Lutz, A. F. Thünemann, H. von Berlepsch and A. Laschewsky, Angew. Chem., Int. Ed., 2005, 44, 5262; (d) Z. Li, M. A. Hillmyer and T. P. Lodge, Nano Lett., 2006, 6, 1245; (e) H. Cui, Z. Chen, S. Zhong, K. L. Wooley and D. J. Pochan, Science, 2007, 317, 647; $(f)$ C. Liu, M. A. Hillmyer and T. P. Lodge, Langmuir, 2008, 24, 12001; $(g)$ S. Yu, T. Azzam, I. Rouiller and A. Eisenberg, J. Am. Chem. Soc., 2009, 131, 10557; $(h)$ F. Schacher, A. Walther, M. Ruppel, M. Drechsler and A. H. E. Müller, Macromolecules, 2009, 42, 3540; ( $i$ ) W. Zhao, D. Chen, Y. Hu, G. M. Grason and T. P. Russell, ACS Nano, 2011, 5, 486; $(j)$ S. J. Holder and N. A. J. M. Sommerdijk, Polym. Chem., 2011, 2, 1018; (k) F. H. Schacher, T. Rudolph, M. Drechsler and A. H. E. Müller, Nanoscale, 2011, 3, 288; (l) P. Chambon, A. Blanazs, G. Battaglia and S. P. Armes, Macromolecules, 2012, 45, 5081; $(m)$ Y. Gao, Y. Wang, M. Jiang and D. Chen, ACS Macro Lett., 2012, 1, 1312; (n) A. O. Moughton, M. A. Hillmyer and T. P. Lodge, Macromolecules, 2012, 45, 2; (o) P. A. Rupar, L. Chabanne, M. A. Winnik and I. Manners, Science, 2012, 337, 559; (p) A. H. Gröschel, A. Walther, T. I. Löbling, F. H. Schacher, H. Schmalz and A. H. E. Müller, Nature, 2013, 503, 247; (q) Y. Matsuo, R. Konno, T. Ishizone, R. Goseki and A. Hirao, Polymers, 2013, 5, 1012; (r) T. I. Löbling, J. S. Haataja, C. V. Synatschke, F. H. Schacher, M. Müller, A. Hanisch, A. H. Gröschel and A. H. E. Müller, ACS Nano, 2014, 8, 11330; (s) T. I. Löbling, O. Borisov, J. S. Haataja, O. Ikkala, A. H. Gröschel and A. H. E. Müller, Nat. Commun., 2016, 7, 12097.
5 (a) K. Takahashi, H. Hasegawa, T. Hashimoto, V. Bellas, H. Iatrou and N. Hadjichristidis, Macromolecules, 2002, 35, 4859; (b) A. K. Brannan and F. S. Bates, Macromolecules, 2004, 37, 8816; (c) A. Takano, K. Soga, T. Asari, J. Suzuki, S. Arai, H. Saka and Y. Matsushita, Macromolecules, 2003, 36, 8216; (d) J. F. Gohy, C. Ott, S. Hoeppener and U. S. Schubert, Chem. Commun., 2009, 6038; (e) A. Hasneen, H. S. Han and H.-J. Paik, React. Funct. Polym., 2009, 69, 681; $(f)$ J. Chen, M. Liu, H. Gong, G. Cui, S. Lü, C. Gao, F. Huang, T. Chen, X. Zhang and Z. Liu, Polym. Chem., 2013, 4, 1815; $(g)$ N. Ekizoglou and N. Hadjichristidis, J. Polym. Sci., Part A: Polym. Chem., 2002, 40, 2166; (h) P. Fragouli, H. Iatrou, D. J. Lohse and N. Hadjichristidis, J. Polym. Sci., Part A: Polym. Chem., 2008, 46, 3938; (i) E. Gungor, G. Hizal and U. Tunca, J. Polym. Sci., Part A: Polym. Chem., 2009, 47, 3409; $(j)$ K. S. Pafiti, C. S. Patrickios, C. Abetz and V. Abetz, J. Polym. Sci., Part A: Polym. Chem., 2013, 51, 4957; (k) H. Liu, K. Miao, G. Zhao, C. Li and Y. Zhao, Polym. Chem., 2014, 5, 3071.

6 (a) G. H. Michler, Electron microscopy of polymers, Springer laboratory manuals in polymer science, Springer, Heidelberg, 2008; (b) F. Nudelman, G. de With and N. A. J. M. Sommerdijk, Soft Matter, 2011, 7, 17; (c) H. Jinnai, T. Tsuchiya, S. Motoki, T. Kaneko, T. Higuchi and A. Takahara, Microscopy, 2013, 62, 243; (d) D. Demurtas, P. Guichard, I. Martiel, R. Mezzenga, C. Hébert and L. Sagalowicz, Nat. Commun., 2015, 6, 8915; (e) W. van Aarle, W. J. Palenstijn, J. De Beenhouwer, T. Altantzis, S. Bals, K. J. Batenburg and J. Sijbers, Ultramicroscopy, 2015, 157, 35; $(f)$ W. van Aarle, W. J. Palenstijn, J. Cant, E. Janssens, F. Bleichrodt, A. Dabravolski, J. De Beenhouwer, K. J. Batenburg and J. Sijbers, Opt. Express, 2016, 24, 25129; $(g)$ W. J. Palenstijn, K. J. Batenburg and J. Sijbers, J. Struct. Biol., 2011, 176, 250; (h) T. L. Jensen, J. H. Jørgensen, P. C. Hansen and S. H. Jensen, BIT Numer. Math., 2012, 52, 329.

7 H. Rullgård, L.-G. Öfverstedt, S. Masich, B. Daneholt and O. Öktem, J. Microsc., 2011, 243, 234.

8 (a) G. J. Clarkson, A. Cook, N. B. McKeown, K. E. Treacher and Z. AliAdib, Macromolecules, 1996, 29, 913; (b) S.-M. Park, S.-H. Yun and B.-H. Sohn, Macromol. Chem. Phys., 2002, 203, 2069.

9 I. A. Ansari and I. W. Hamley, J. Mater. Chem., 2003, 13, 2412.

10 (a) J. S. Trent, J. I. Scheinbeim and P. R. Couchman, Macromolecules, 1983, 16, 589; (b) M. Vayer, T. H. Nguyen and C. Sinturel, Polymer, 2014, 55, 1048; (c) R. Olayo-Valles, M. S. Lund, C. Leighton and M. A. Hillmyer, J. Mater. Chem., 2004, 14, 2729; (d) M. Shoji, M. Eguchi, J. M. Layman, M. P. Cashion, T. E. Long and H. Nishide, Macromol. Chem. Phys., 2009, 210, 579; $(e)$ Y. Wang, N. Coombs, A. Turak, Z.-H. Lu, I. Manners and M. A. Winnik, Macromolecules, 2007, 40, 1594.

11 (a) P. A. Beales, J. Nam and T. K. Vanderlick, Soft Matter, 2011, 7, 1747; (b) G. Milosevits, Z. Rozsnyay, G. T. Kozma, J. Milosevits, G. Tömöry, H. Robotka, L. Rosivall and J. Szebenia, Chem. Phys. Lipids, 2012, 165, 482. 